\title{
A message from the new editor-in-chief
}

\author{
Noriomi Matsumura ${ }^{1}$
}

Published online: 27 March 2020

(c) The Japan Society of Clinical Oncology 2020

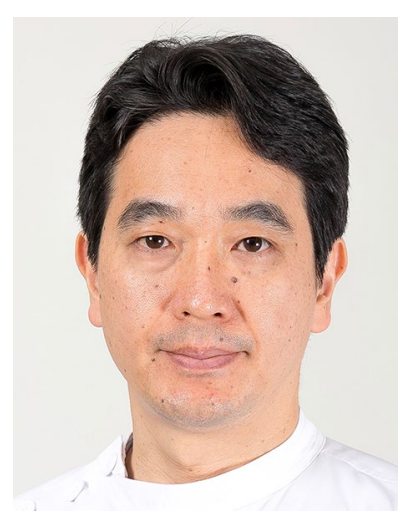

It is a great honor for me to be appointed as the new editor-in chief of the International Cancer Conference Journal (ICCJ) effective February 2020. ICCJ, founded in 2012, is an official journal of the Japan Society of Clinical Oncology (JSCO).

This journal publishes original case reports on all types of cancer. In cancer treatment, especially in cases of rare tumors or cases that are difficult to treat, it is important to carefully treat each one separately, with experts from various fields discussing it in the form of a "cancer conference." The purpose of this journal is to promote personalized treatment by publishing such case reports.

Advances in the effective treatment of rare diseases and conditions, and the development of new surgical techniques and pharmacotherapies, are needed in order to promote individualized cancer treatment. Furthermore, treatment based on molecular characterization, also known as precision medicine, has recently gained in prominence. For molecular characterization, immunohistochemical staining has been performed for some time. In addition, it has

Noriomi Matsumura

noriomi@med.kindai.ac.jp

1 Department of Obstetrics and Gynecology, Faculty of Medicine, Kindai University, Osakasayama, Japan become common practice to examine gene amplification by fluorescent in situ hybridization and to examine oncogenic mutation by sequencing analysis to determine the adaptation of molecular targeted drugs. More recently, rapid advances in sequencing technology have made gene panel testing possible, which allows hundreds of gene mutations and fusion genes to be examined simultaneously. Moreover, genetic panel testing can be used not only to administer approved molecularly targeted drugs, but also to enroll subjects in clinical trials, and to detect many non-druggable gene mutations. In the future, with the advancement of technology, omics analysis including exome sequencing and transcriptome analysis will be widely performed clinically, allowing us to detect many gene alterations whose clinical significance was unknown at the time of testing.

The scope of this journal includes all case reports that can potentially support personalized cancer treatment, and is not necessarily limited to those that underwent molecular analysis. However, we particularly welcome reports on molecularly analyzed cancer cases, including gene mutations, gene fusions, gene expression, and changes in copy number, regardless of their known clinical significance. Assessing the molecular analysis of a tumor usually requires a "cancer conference" where experts from various fields discuss it. Even if the authors and their respective "cancer conference" were unable to determine the clinical significance of molecular changes at the time of submission and publication, the data may provide evidence that will help the scientific community develop precision medicine solutions in the future.

Publisher's Note Springer Nature remains neutral with regard to jurisdictional claims in published maps and institutional affiliations. 